\title{
Hydrodynamic attractor of a hybrid viscous fluid in Bjorken flow
}

\author{
Toshali Mitra, ${ }^{1, *}$ Sukrut Mondkar, ${ }^{2, \dagger}$ Ayan Mukhopadhyay $\bullet,{ }^{2, \ddagger}$ Anton Rebhan, ${ }^{3, \S}$ and Alexander Soloviev $๑^{4, \|}$ \\ ${ }^{1}$ The Institute of Mathematical Sciences, Chennai 600113, India \\ ${ }^{2}$ Department of Physics, Indian Institute of Technology Madras, Chennai 600036, India \\ ${ }^{3}$ Institut für Theoretische Physik, Technische Universität Wien, Wiedner Hauptstrasse 8-10, A-1040 Vienna, Austria \\ ${ }^{4}$ Department of Physics and Astronomy, Stony Brook University, Stony Brook, New York 11794, USA
}

(Received 22 June 2020; accepted 12 November 2020; published 4 December 2020)

\begin{abstract}
The nonequilibrium evolution in a boost-invariant Bjorken flow of a hybrid viscous fluid model containing two interacting components with different viscosities, such that they represent strongly and weakly self-coupled sectors, is shown to be characterized by a hydrodynamic attractor which has an early-time behavior that is reminiscent of the so-called bottom-up thermalization scenario in heavy-ion collisions. The hydrodynamization times for the two sectors can differ strongly, with details depending on the curve realized on the two-dimensional attractor surface, which might account for different scenarios for small and large systems in nuclear collisions. The total system behaves as a single viscous fluid with a dynamically determined effective shear viscosity.
\end{abstract}

DOI: 10.1103/PhysRevResearch.2.043320

\section{INTRODUCTION}

Hydrodynamic models have enjoyed astounding success in describing the collective flow of low- $p_{T}$ hadrons in heavy-ion collisions [1-5]. These models require hydrodynamics to be initialized at $\lesssim 1 \mathrm{fm} / \mathrm{c}$ after the collisions when the system is far away from equilibrium and with a very low ratio of shear viscosity to entropy density $(\eta / s \lesssim 0.2)$. The studies of heavy-ion collisions in strongly interacting gauge theories using holographic methods [6,7] were the first to provide theoretical insights not only into the low ratio of shear viscosity to entropy density $[4,8]$, but also into the applicability of hydrodynamics itself in far away from equilibrium conditions. In particular, it was understood that the hydrodynamic expansion (which generically has a zero radius of convergence) needs to be resummed to all orders in derivatives in order to generate a causal evolution which can be matched with the evolution in the exact microscopic theory for an arbitrary initial condition [9-11]. Furthermore, the resummed hydrodynamic expansion can be expressed as an appropriate trans-series from which one could also extract the quasinormal-type relaxation modes of the system.

Subsequently, it was established that a wide variety of other phenomenological approaches, such as Müller-Israel-Stewart

\footnotetext{
*toshalim@imsc.res.in

†sukrutmondkar@gmail.com

‡ayan@physics.iitm.ac.in

§anton.rebhan@tuwien.ac.at

"alexander.soloviev@ stonybrook.edu
}

Published by the American Physical Society under the terms of the Creative Commons Attribution 4.0 International license. Further distribution of this work must maintain attribution to the author(s) and the published article's title, journal citation, and DOI.
(MIS) $[12,13]$ and related versions of extended hydrodynamics $[10,14,15]$, and kinetic theory [15-19] also demonstrated the existence of a hydrodynamic attractor, which is the evolution obtained from resumming the hydrodynamic expansion to all orders and to which the system approaches rather quickly for any initial condition. The trans-series has been also examined in such contexts, notably in Refs. [10,14,20-22]. The ubiquitous presence of a hydrodynamic attractor provides a stronger foundation for hydrodynamics as a causal and consistent effective theory that may be applied generally even when the system is yet to achieve local equilibration. The approach to the hydrodynamic attractor provides a more precise meaning to hydrodynamization [7,23] of the system, referring to the feature that the energy-momentum tensor and conserved currents can be described by an effective hydrodynamic theory very accurately after a time that is commensurate with a microscopic timescale for any arbitrary initial data (see Ref. [24] for a nice discussion). Strong coupling leads to more rapid hydrodynamization [10] with significant qualitative differences from weak-coupling scenarios [25].

A key feature of quantum chromodynamics (QCD) is asymptotic freedom, which in ultrarelativistic heavy-ion collisions makes it possible to describe hard quasiparticle degrees of freedom to some extent by perturbative QCD (in terms of the glasma effective theory [26]) and kinetic theory. However, the gluons produced in their interactions have a coupling that is the stronger the smaller their momenta are, so that strong-coupling methods are needed for the description of the developing bath of soft gluons.

In the so-called bottom-up thermalization scenario initially proposed in Ref. [27] and refined in Ref. [28], isotropization and eventually thermalization results from the buildup of a thermal bath of soft gluons. This is in contrast to the completely strong-coupling picture provided by a gauge/gravity duality, where thermalization is instead top-down [29]. In Refs. [30,31] it was attempted to find a transition from top- 
down to bottom-up scenarios as the infinite coupling limit of the standard anti-de Sitter (AdS)/conformal field theory (CFT) correspondence is relaxed through higher-curvature corrections [32]. However, since the quark-gluon medium produced in heavy-ion collisions involves more strongly and more weakly coupled sectors simultaneously, a hybrid approach which combines weak- and strong-coupling features may be required.

To this end, in Refs. [33-35] the semiholographic approach originally developed by Refs. [36,37] in the context of nonFermi liquids was utilized for combining the weak-coupling glasma framework [26] with a holographic AdS/CFT description of the infrared sector of soft gluons. ${ }^{1}$ In more general terms, a hybrid two-fluid system with couplings analogous to those used in the semiholographic approach was introduced and studied in Ref. [39] in thermodynamic equilibrium.

this paper we present the results of a study of the nonequilibrium evolution of the hybrid fluid model of Ref. [39] in a boost-invariant longitudinal expansion known as Bjorken flow [40]. The two components are assumed to have a conformal equation of state, but different amounts of shear viscosity and relaxation times, chosen so that one component corresponds to the infinite coupling limit in AdS/CFT [41] and the other has significant higher shear viscosity corresponding to a less strongly coupled fluid. The two fluids can exchange energy and momentum through mutual deformations of an effective metric that the respective subsystems are living in, while the resulting total energy-momentum tensor of the full system (which actually lives in Minkowski space) is conserved, but with a nonvanishing trace caused by the interactions.

The nonequilibrium evolution of the full system turns out to involve a hydrodynamic attractor, which defines a two-dimensional hypersurface in the four-dimensional ${ }^{2}$ phase space spanned by the degrees of freedom of the subsystems. Generically, we find that the attractor requires an initial evolution of the distribution of energy in the subsystems that is reminiscent of the bottom-up thermalization scenario, where the energy is dominated by the more viscous (weakly selfcoupled) sector at early times and rapidly shared with the less viscous (strong-coupling) sector.

Subsequently, significant differences in the hydrodynamization times of the subsystems are observed, after which the energy-momentum tensor of the full system can be described hydrodynamically with a specific viscosity determined by which curve on the attractor hypersurface the system follows.

We want to emphasize, however, that although our model here is inspired from semiholography, it does not capture all the complexities of the latter. We mainly borrow the idea of democratic couplings between the effective descriptions of the perturbative and nonperturbative sectors, which have been shown to pass stringent consistency checks with the princi-

\footnotetext{
${ }^{1}$ For another hybrid approach, see Ref. [38], which was aimed at the description of the energy loss of hard jets moving through a strongly coupled medium.

${ }^{2}$ The symmetries of the effectively $1+1$-dimensional Bjorken flow admit different longitudinal and transverse pressures for each of the two (conformal) subsystems.
}

ples of thermodynamics, statistical mechanics, and Wilsonian renormalization group flow $[39,42]$. While a semiholographic description involving a similar coupling between a kinetic sector and a dynamical black hole will exhibit irreversible energy transfer to the soft sector on longer timescales, as seen in Ref. [35], in our two-fluid setup there is no such mechanism. Our model can therefore capture only certain aspects of the evolution described by a semiholographic setup (also the description of the early stages of the weakly coupled sector as a fluid is a drastic simplification). A noteworthy feature of our model is that the full system can still be described hydrodynamically at late times even when local equilibration cannot happen due to a lack of sufficient time for mutual interactions between its components.

Besides its intrinsic interest as a tractable two-fluid model with a hydrodynamic attractor, which has many possibilities of generalizations, our model is of specific interest to quarkgluon plasma physics, where the interplay of hard and soft sectors of the dynamics can be modeled in a different way. While it is clear that it cannot capture the specific interactions described by QCD, it might reveal features of the nonequilibrium dynamics of QCD that have a more general character. For instance, the observed dependence of the ratio of hydrodynamization times on the extra parameters of the attractor surface might account for different scenarios for small and large systems in nuclear collisions.

\section{HYBRID FLUID MODEL WITH BJORKEN FLOW}

In Ref. [39] a minimal coupling of the energy-momentum tensors of two subsystems was introduced in the following, purely geometrical way, inspired by the semiholographic setup of Refs. [33-35,42]. The combined system is defined on one and the same spacetime with metric tensor $g_{\mu \nu}^{(\mathrm{B})}$, but the two energy-momentum tensors of the subsystems, denoted by $t^{\mu \nu}$ and $\tilde{t}^{\mu \nu}$, are assumed to be covariantly conserved only with respect to effective metric tensors $g_{\mu \nu}$ and $\tilde{g}_{\mu \nu}$, which differ from $g_{\mu \nu}^{(\mathrm{B})}$ and are determined locally by the respective other subsystem according to

$$
\begin{aligned}
& g_{\mu \nu}=g_{\mu \nu}^{(\mathrm{B})}+\frac{\sqrt{-\tilde{g}}}{\sqrt{-g^{(\mathrm{B})}}}\left[\gamma \tilde{t}^{\alpha \beta} g_{\alpha \mu}^{(\mathrm{B})} g_{\beta \nu}^{(\mathrm{B})}+\gamma^{\prime} \operatorname{tr}\left(\tilde{t} \cdot g^{(\mathrm{B})}\right) g_{\mu \nu}^{(\mathrm{B})}\right], \\
& \tilde{g}_{\mu \nu}=g_{\mu \nu}^{(\mathrm{B})}+\frac{\sqrt{-g}}{\sqrt{-g^{(\mathrm{B})}}}\left[\gamma t^{\alpha \beta} g_{\alpha \mu}^{(\mathrm{B})} g_{\beta \nu}^{(\mathrm{B})}+\gamma^{\prime} \operatorname{tr}\left(t \cdot g^{(\mathrm{B})}\right) g_{\mu \nu}^{(\mathrm{B})}\right],
\end{aligned}
$$

with two coupling constants $\gamma, \gamma^{\prime} \equiv-r \gamma$ with mass dimension -4 . (We need $\gamma>0$ in order that the dynamics of the subsystems remains causal with respect to the physical background metric, and $r>1$ for UV completeness [39].) These coupling rules ensure that the full system has a conserved energy-momentum tensor in the physical background, $\nabla_{\mu}^{(\mathrm{B})} T^{\mu}{ }_{\nu}=0$, with

$$
\begin{aligned}
T_{v}^{\mu}= & \frac{1}{2}\left[\left(t^{\mu}{ }_{v}+t_{v}{ }^{\mu}\right) \frac{\sqrt{-g}}{\sqrt{-g^{(\mathrm{B})}}}+\left(\tilde{t}^{\mu}{ }_{v}+\tilde{t}_{v}{ }^{\mu}\right) \frac{\sqrt{-\tilde{g}}}{\sqrt{-g^{(\mathrm{B})}}}\right] \\
& +\Delta K \delta_{v}^{\mu}=: T_{1 v}^{\mu}\left(\mathcal{E}_{1}, \mathcal{P}_{1}\right)+T_{2}^{\mu}{ }_{v}\left(\mathcal{E}_{2}, \mathcal{P}_{2}\right)+T^{\mu}{ }_{v, \text { int }},
\end{aligned}
$$


where

$$
\begin{aligned}
\Delta K= & -\frac{\gamma}{2}\left(t^{\rho \alpha} \frac{\sqrt{-g}}{\sqrt{-g^{(\mathrm{B})}}}\right) g_{\alpha \beta}^{(\mathrm{B})}\left(\tilde{t}^{\beta \sigma} \frac{\sqrt{-\tilde{g}}}{\sqrt{-g^{(\mathrm{B})}}}\right) g_{\sigma \rho}^{(\mathrm{B})} \\
& -\frac{\tilde{\gamma}}{2}\left(t^{\alpha \beta} g_{\alpha \beta}^{(\mathrm{B})} \frac{\sqrt{-g}}{\sqrt{-g^{(\mathrm{B})}}}\right)\left(\tilde{t}^{\sigma \rho} g_{\sigma \rho}^{(\mathrm{B})} \frac{\sqrt{-\tilde{g}}}{\sqrt{-g^{(\mathrm{B})}}}\right) .
\end{aligned}
$$

In the Bjorken-flow case in flat Minkowski spacetime, with a boost-invariant transversely homogeneous expansion in the $z$ direction, the background metric will be given in Milne coordinates $(\tau, x, y, \zeta)$ where $g_{\mu \nu}^{(\mathrm{B})}=\operatorname{diag}\left(-1,1,1, \tau^{2}\right)$ with proper time $\tau=\sqrt{t^{2}-z^{2}}$ and spacetime rapidity $\zeta=$ $\tanh ^{-1}(z / t)$. In this situation, a boost-invariant ansatz for the effective metric tensors of the subsystems is given by six scalar functions of $\tau$ according to

$$
\begin{aligned}
& g_{\mu \nu}(\tau)=\operatorname{diag}\left(-a^{2}, b^{2}, b^{2}, c^{2}\right), \\
& \tilde{g}_{\mu \nu}(\tau)=\operatorname{diag}\left(-\tilde{a}^{2}, \tilde{b}^{2}, \tilde{b}^{2}, \tilde{c}^{2}\right),
\end{aligned}
$$

and the coupling equations (1) provide six nonlinear algebraic equations to solve for them.

The equations of state of the subsystems are assumed to be conformal, $\epsilon=3 P, \tilde{\epsilon}=3 \tilde{P}$, so that the ansatz for the energymomentum tensors of the subsystem reads

$$
\begin{aligned}
t^{\mu \nu} & =\operatorname{diag}\left(\frac{\varepsilon}{a^{2}}, \frac{P_{\perp}}{b^{2}}, \frac{P_{\perp}}{b^{2}}, \frac{P_{L}}{c^{2}}\right) \\
& =\operatorname{diag}\left(\frac{\varepsilon}{a^{2}}, \frac{P}{b^{2}}, \frac{P}{b^{2}}, \frac{P}{c^{2}}\right)+\pi^{\mu \nu}, \\
\pi^{\mu \nu} & =\operatorname{diag}\left(0, \frac{\phi}{2 b^{2}}, \frac{\phi}{2 b^{2}},-\frac{\phi}{c^{2}}\right),
\end{aligned}
$$

and analogously for $\tilde{t}^{\mu \nu}$. Notice, however, that the full system (2) is not conformal, but has a nonvanishing trace of the energy-momentum tensor produced by the interactions of the two subsystems, which involve the dimensionful coupling $\gamma$, whose scale could be set by the saturation momentum $Q_{s}$ of the glasma effective theory [26].

Covariant conservation with respect to the effective metric (but not with respect to the physical background metric) $\nabla_{\mu} t^{\mu \nu}=0$ yields

$$
\partial_{\tau} \varepsilon+\varepsilon \partial_{\tau} \log \left(b^{8 / 3} c^{4 / 3}\right)+\phi \partial_{\tau} \log b / c=0 .
$$

The dynamical system is closed by assuming relaxation of $\pi^{\mu \nu}$ by MIS equations with shear viscosities $\eta, \tilde{\eta}$ and relaxation times $\tau_{\pi}, \tilde{\tau}_{\pi}$ (cf. Ref. [41]),

$$
\left(\tau_{\pi} u^{\alpha} \nabla_{\alpha}+1\right) \pi^{\mu \nu}=-\eta \sigma^{\mu \nu},
$$

yielding

$$
\tau_{\pi} \partial_{\tau} \phi+\frac{4}{3} \eta \partial_{\tau} \log (b / c)+\left[a+\frac{4}{3} \tau_{\pi} \partial_{\tau} \log \left(b^{2} c\right)\right] \phi=0,
$$

and similarly for the other subsystem. In the following we parametrize $\eta=C_{\eta}(\epsilon+P) / T$ and $\tau_{\pi}=C_{\tau} / T$ with dimensionless constants $C_{\eta}$ and $C_{\tau}$, where for simplicity we identify $T \equiv \epsilon^{1 / 4}$. Causality requires that $C_{\tau}>2 C_{\eta}$ [4].

In the following we choose the first system to have ten times higher specific viscosity than the second, $C_{\eta}=10 \tilde{C}_{\eta}$.
Having in mind subsystems that are governed by kinetic theory and AdS/CFT, respectively, we set [4] $C_{\tau}=5 C_{\eta}$ and $\tilde{C}_{\eta}=1 /(4 \pi), \tilde{C}_{\tau}=(2-\ln 2) /(2 \pi)$. (Note that in contrast to kinetic theory, our fluid model does not require $P_{\perp}$ and $P_{L}$ to be both positive definite.)

\section{TWO-FLUID ATTRACTOR}

As found in Refs. [10,43], a single conformal fluid described by the two equations (8) and (10) has a hydrodynamic attractor that can be characterized by the initial condition

$$
\lim _{\tau \rightarrow 0} \chi(\tau)=\sqrt{\frac{C_{\eta}}{C_{\tau}}}=: \sigma,
$$

where

$$
\chi:=\frac{\phi}{\epsilon+P} .
$$

[In Ref. [21], $\mathcal{A}=\left(P_{\perp}-P_{L}\right) / P$ was defined as a measure of anisotropy, which for a conformal fluid is proportional to $\chi$ : $\mathcal{A}=6 \chi$. Note also that for a conformal fluid $\chi \geqslant 1 / 4$ implies $P_{L} \leqslant 0$.] Generic solutions apart from the attractor solution are either singular at finite $\tau$ or have negative $\chi$ at early times with a limiting value $-\sigma$ at $\tau=0$. Solutions which are regular for all $\tau>0$ have positive $\epsilon$ throughout, with $\epsilon$ diverging as $\tau \rightarrow 0$.

When the two fluids are coupled according to (1), a common attractor arises where each fluid still has (11) as a limiting value, but the behavior of the energy densities is strongly modified. With $0<\sigma<\tilde{\sigma}<1 / \sqrt{2}$, as is the case for our choice of parameters $(\sigma \approx 0.45, \tilde{\sigma} \approx 0.62)$, it turns out that for the common attractor $\epsilon$ always approaches the finite value $\sqrt{(r-1) / r} \gamma^{-1}$ and $\tilde{\epsilon}$ vanishes as $\tau \rightarrow 0$. But these quantities are defined with respect to the effective metric tensors $g$ and $\tilde{g}$ which become singular as $\tau \rightarrow 0$. Viewed from the flat Minkowski-Milne background, the contribution to the total energy density from the first system is

$$
\mathcal{E}_{1}:=\left(a b^{2} c / \tau\right) \epsilon \sim \tau^{4(\sigma-1) / 3},
$$

and thus diverging for $\tau \rightarrow 0$ as the single-fluid case. With $\tilde{\sigma}>\sigma$, the contribution of the second, less viscous fluid is suppressed at early times as

$$
\mathcal{E}_{2} / \mathcal{E}_{1} \sim \tau^{8(\tilde{\sigma}-\sigma) / 3} .
$$

(Depending on the parameters $\sigma$ and $\tilde{\sigma}, \mathcal{E}_{2}$ can diverge or go to zero as $\tau \rightarrow 0$; for the above choice of parameters it also diverges, but less strongly than $\mathcal{E}_{1}$.)

This initial distribution of the energy densities is reminiscent of the bottom-up scenario of thermalization in heavy-ion collisions [27,28], where the evolution starts out with the total energy density concentrated in the more weakly coupled sector, which then gets redistributed to the more strongly coupled soft degrees of freedom.

Away from the limit $\tau \rightarrow 0$, the differential equations and the nonlinear algebraic coupling equations can only be solved numerically.

In Fig. 1 we display one particular attractor solution and neighboring trajectories in a plot of the anisotropy variables $\chi$, $\tilde{\chi}$, and $\chi_{\text {tot }}$ of the individual subsystems and the total system. 


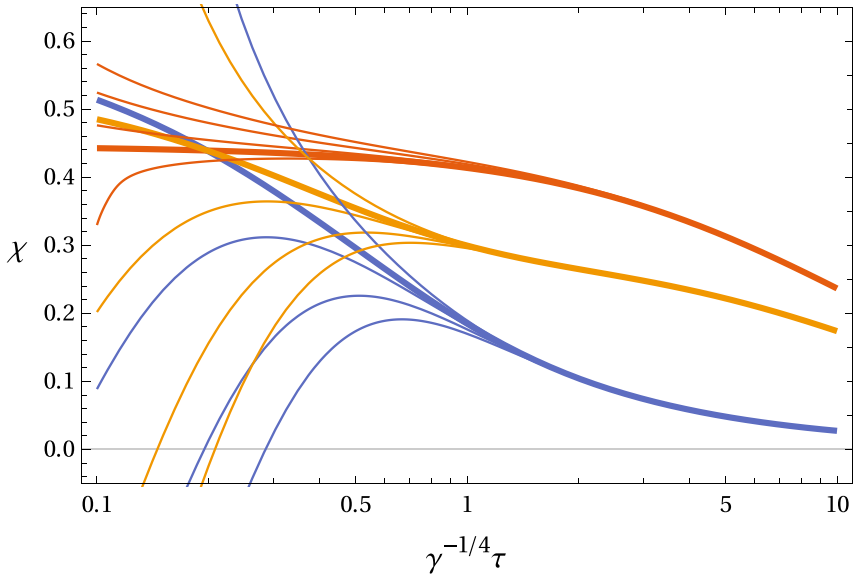

FIG. 1. $\chi$ 's of the attractor solution of Fig. 2 (thick lines) and four neighboring trajectories (thin lines). The less viscous (strongcoupling, "soft") system is displayed by blue curves, the more viscous ("hard") system by red curves, and the total system by orange curves.

The complete set of attractor solutions is in fact a twodimensional manifold which can be parametrized by the dimensionless energy densities of the subsystems $\gamma \epsilon$ and $\gamma \tilde{\epsilon}$ at some nonzero reference time.

The attractor surface also depends on $r \equiv-\gamma^{\prime} / \gamma>1$. When $r$ is sufficiently close to 1 , the two-fluid system exhibits a first-order phase transition (with a second-order endpoint at $r=r_{c}$ ) as analyzed in detail in Ref. [39]. Here, we have chosen $r=2$ so that there is only a crossover behavior during the evolution of the system, as is indeed the case for QCD for high temperature and small quark chemical potential.

In Fig. 2 the particular attractor solution in Fig. 1 is displayed in more detail. In the left panel, the evolutions of the energy densities $\mathcal{E}_{1}, \mathcal{E}_{2}, \mathcal{E}_{\text {total }}$ are shown for initial conditions which lead to $\mathcal{E}_{1}=\mathcal{E}_{2}$ at $\gamma^{-1 / 4} \tau=1$ and more energy in the less viscous system for a stretch of time before.

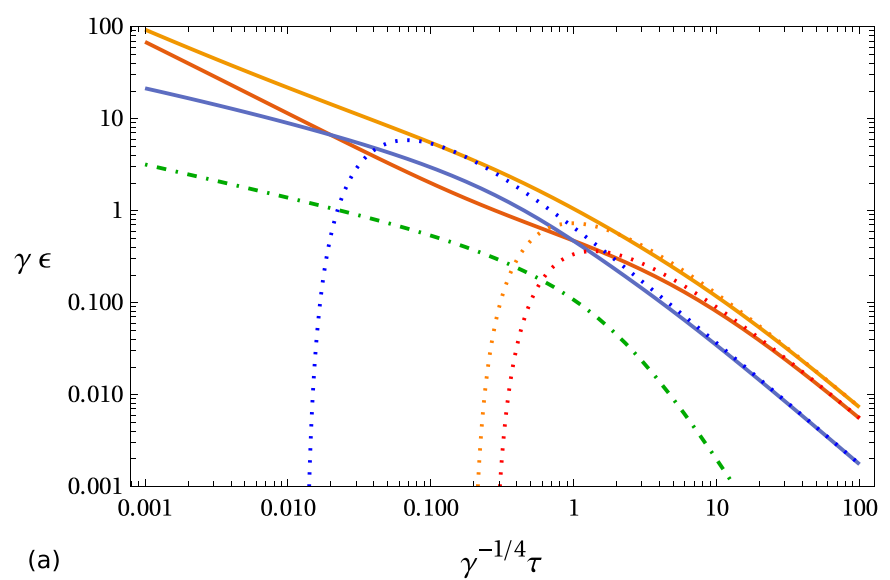

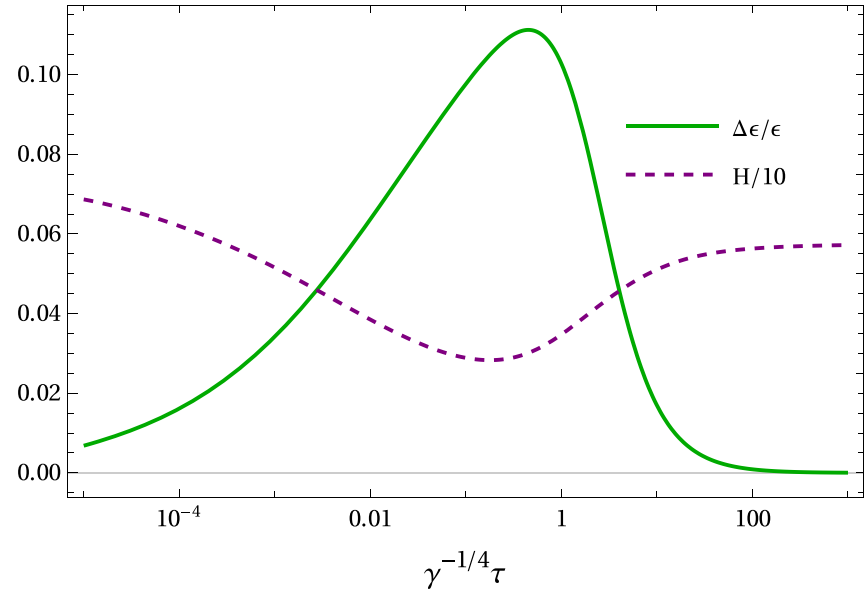

FIG. 3. Interaction energy between the two subsystems over total energy (solid green line), which is proportional to the trace of the total energy-momentum tensor, and the averaged shear viscosity $\mathrm{H}$ (dashed purple line) as defined in (15) - the latter rescaled to fit in the same plot.

Other attractor solutions exist where $\mathcal{E}_{2}$ exceeds $\mathcal{E}_{1}$ for a longer time or where $\mathcal{E}_{2}$ never reaches $\mathcal{E}_{1}$. However, common to all is, as already explained, that at early times the initial energy is concentrated in the more viscous ("hard") sector which during the Bjorken expansion is gradually transferred to the less viscous ("soft") sector and to the interaction energy of the two subsystems (the latter is also displayed in Fig. 2 and as a ratio in a linear plot in Fig. 3). As the system cools down towards its crossover regime, the relative amount of interaction energy rises and then switches off rapidly. Subsequently the two subsystems as well as the total system all approach perfect-fluid behavior $\epsilon \sim \tau^{-4 / 3}$. Here, any similarity to heavy-ion collisions of course ends, which instead ends with the free-streaming of hadrons. At larger times a large fraction of the energy is transferred back to the more weakly interacting sector. Although this is reminiscent of the

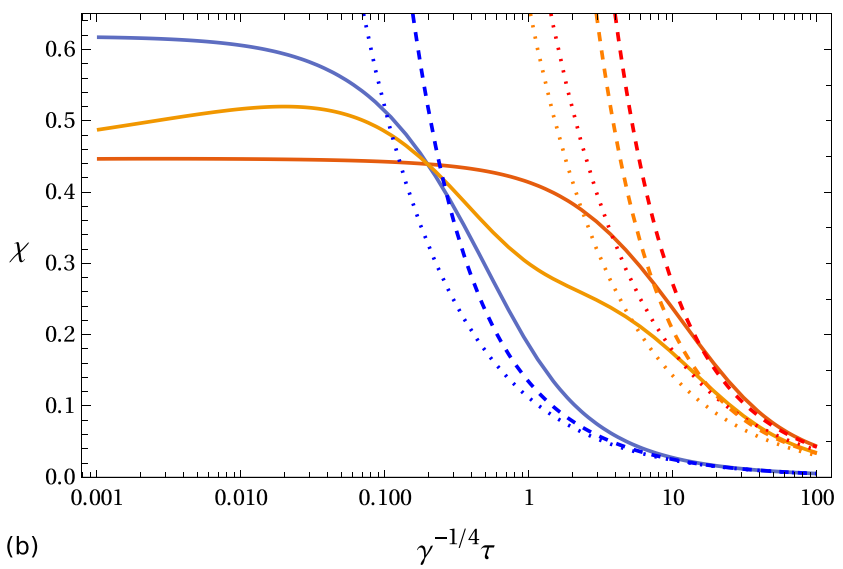

FIG. 2. (a) Energy densities and (b) $\chi$ 's of one particular attractor curve where the initially subdominant energy density in the less viscous ("soft") system grows larger than that in the initially dominant more viscous ("hard") system (blue and red curves, respectively; total system represented by orange curves). The green dashed-dotted line gives the interaction energy between the two subsystems. In both plots, the dotted lines correspond to a first-order hydrodynamics approximation; in the $\chi$ plots also the second-order hydrodynamics approximation is shown (dashed lines). 
TABLE I. Subsystem hydrodynamization times and the concurrent values of the dimensionless quantities $w=\mathcal{E}_{1}^{1 / 4} \tau, \tilde{w}=\mathcal{E}_{2}^{1 / 4} \tau, \chi$, and $\tilde{\chi}$ for three scenarios with different values of $\mathcal{E}_{1}(1)=\mathcal{E}_{2}(1)=$ : $\mathcal{E}(1)$, where all dimensionful quantities are given in units of $\gamma$. The last column gives the ratio $R_{\mathrm{hd}}:=\tau_{\mathrm{hd}} / \tilde{\tau}_{\mathrm{hd}}$.

\begin{tabular}{lccccccc}
\hline \hline $\mathcal{E}(1)$ & $\tau_{\text {hd }}$ & $w_{\text {hd }} / 10$ & $\chi_{\text {hd }}$ & $\tilde{\tau}_{\text {hd }}$ & $\tilde{w}_{\text {hd }}$ & \multicolumn{1}{c}{$\tilde{\chi}_{\text {hd }}$} & $R_{\text {hd }}$ \\
\hline 0.26 & 12.0 & 0.609 & 0.215 & 2.08 & 1.42 & 0.101 & 5.76 \\
0.32 & 10.2 & 0.705 & 0.203 & 3.90 & 2.82 & 0.0525 & 2.62 \\
0.052 & 25.5 & 0.608 & 0.210 & 1.39 & 0.613 & 0.211 & 18.4 \\
\hline \hline
\end{tabular}

transition to a more weakly interacting hadron gas after the crossover transition of QCD, it is clear that our model can be considered as a toy model for heavy-ion collisions only with regard to certain aspects. Also, the boost-invariant Bjorken flow is only relevant for its early stages.

In the right panel of Fig. 2, the evolution of anisotropy in the subsystems and in the full system are displayed. While the subsystems show a monotonic decrease of anisotropy, this is not the case for the full system, where $\chi_{\text {tot }}$ interpolates the values in the subsystems in a more complicated manner.

From an analysis of the asymptotic behavior, which can be performed analytically and will be discussed in more detail elsewhere, one can show that the full system behaves as a single viscous fluid with an effective shear viscosity given by

$$
C_{\eta}^{\mathrm{eff}}=\lim _{\tau \rightarrow \infty} \mathrm{H}(\tau)=\lim _{\tau \rightarrow \infty} \frac{C_{\eta} \epsilon^{4 / 3}(\tau)+\tilde{C}_{\eta} \tilde{\epsilon}^{4 / 3}(\tau)}{[\epsilon(\tau)+\tilde{\epsilon}(\tau)]^{4 / 3}},
$$

with the function $\mathrm{H}$ displayed in Fig. 3 (showing a perhaps fortuitous resemblance to the expected behavior of the shear viscosity across the transition from the deconfined phase of QCD to a hadron gas).

In both plots of Fig. 2, the results are compared with firstorder hydrodynamics approximations; in the plots of $\chi$ 's, also the second-order approximations are shown. This comparison allows one to define hydrodynamization times. In order to be close to previous definitions [21,23], we require

$$
\frac{\left|\Delta P_{L}\right|}{P}:=\frac{\left|\phi-\phi_{1 \mathrm{st}}\right|}{P}<0.1 \text { for } \tau>\tau_{\mathrm{hd}}
$$

and similarly for the second subsystem. For the particular solution in Fig. 2 we obtain the ratio $R_{\text {hd }}:=\tau_{\text {hd }} / \tilde{\tau}_{\text {hd }} \approx 5.76$. As one might expect, the hydrodynamization time is longer for the hard sector than for the soft, more strongly interacting sector. In Table I, these times are given in units of $\gamma^{1 / 4}$ and also converted to the dimensionless quantities $w=\mathcal{E}_{1}^{1 / 4} \tau$, $\tilde{w}=\mathcal{E}_{2}^{1 / 4} \tau$. The latter roughly scale according to $4 \pi \eta / s$, albeit with some difference in the second subsystem, reflecting the fact that at $\tau=\tilde{\tau}_{\text {hd }}$ there is still an important exchange of energy with the first, not yet hydrodynamized, subsystem. Also given are the values of $\chi$ at the point of hydrodynamization, showing that the more viscous hard sector is still strongly anisotropic (with $P_{L} / P \equiv 1-4 \chi \approx 0.14$ ) at $\tau=\tau_{\text {hd }}$, while the soft sector is somewhat closer to isotropy (with $\tilde{P}_{L} / \tilde{P} \approx$ 0.6) at the earlier time $\tau=\tilde{\tau}_{\text {hd }}$.
This ordering is rather generic, ${ }^{3}$ but the ratio $R_{\mathrm{hd}}$ depends strongly on the initial conditions. In order to illustrate this, two further cases are also shown in Table I. When the total energy in the system at $\gamma^{-1 / 4} \tau=1$ where we have set $\mathcal{E}_{1}=\mathcal{E}_{2}$ is increased until we reach the limits of the attractor hypersurface, the ratio is reduced to $R_{\mathrm{hd}} \approx 2.62$. Conversely, if the total energy at this reference point is made as small as possible, the ratio becomes $R_{\mathrm{hd}} \approx 18.4$. This dependence on the extra parameters of the attractor hypersurface could perhaps be interpreted as a hint that the hydrodynamic evolution of so-called small systems in nuclear collisions (high multiplicity events in $p-A$ and $p$ - $p$ collisions) [44-47] may involve a markedly different hydrodynamization scenario than the larger systems produced in heavy-ion collisions.

A more complete exploration of the possible evolutions of the hybrid fluid system presented here as well as a study of the details of how a general solution decays onto the attractor will be the subject of a longer publication.

To conclude, we believe to have demonstrated that the hybrid fluid model introduced in Ref. [39], where its equilibrium properties have been analyzed, in combination with MIS equations, provides an interesting model for the nonequilibrium dynamics of a two-component system with different amounts of self-interactions and transport coefficients. Clearly, many generalizations of this model can be entertained. For example, with a smaller parameter $r$ one can also include first-order phase transitions, and the equation of state of the subsystems need not be conformal.

In the special application to boost-invariant (Bjorken) flow $^{4}$ considered here, we have found an intriguing model of bottom-up thermalization, which has been proposed in the context of perturbative QCD [27,28]. Remarkably, this scenario turned out to be a universal feature of our model, if $\tilde{\sigma}>\sigma$, as is the case for a strongly coupled sector with transport coefficients as given by AdS/CFT on the one hand and a weakly coupled sector with larger specific shear viscosity on the other hand.

\section{ACKNOWLEDGMENTS}

A.M. acknowledges support from the Ramanujan Fellowship and ECR award of the Department of Science and Technology of India and also the New Faculty Seed Grant of IIT Madras. A.S. is supported by the Austrian Science Fund (FWF), Project No. J4406.

\footnotetext{
${ }^{3}$ We have also managed to find counterexamples, but only in extreme choices of initial conditions where the soft sector is so suppressed at all times that it hardly contributes to the total energymomentum tensor.

${ }^{4}$ Recently, the identification of the hydrodynamic attractor using the principal component analysis in evolutions that are more complex than the Bjorken flow has been discussed in Ref. [48]. It would also be interesting to extend these kinds of studies to the case of the hybrid fluid considered here.
} 
[1] U. Heinz and R. Snellings, Collective flow and viscosity in relativistic heavy-ion collisions, Annu. Rev. Nucl. Part. Sci. 63, 123 (2013).

[2] C. Gale, S. Jeon, and B. Schenke, Hydrodynamic modeling of heavy-ion collisions, Int. J. Mod. Phys. A 28, 1340011 (2013).

[3] W. Florkowski, M. P. Heller, and M. Spalinski, New theories of relativistic hydrodynamics in the LHC era, Rep. Prog. Phys. 81, 046001 (2018).

[4] P. Romatschke and U. Romatschke, Relativistic Fluid Dynamics In and Out of Equilibrium, Cambridge Monographs on Mathematical Physics (Cambridge University Press, Cambridge, UK, 2019).

[5] J. Berges, M. P. Heller, A. Mazeliauskas, and R. Venugopalan, Thermalization in QCD: Theoretical approaches, phenomenological applications, and interdisciplinary connections arXiv:2005.12299.

[6] P. M. Chesler and L. G. Yaffe, Holography and Colliding Gravitational Shock Waves in Asymptotically $\mathrm{AdS}_{5}$ Spacetime, Phys. Rev. Lett. 106, 021601 (2011).

[7] M. P. Heller, R. A. Janik, and P. Witaszczyk, Characteristics of Thermalization of Boost-Invariant Plasma from Holography, Phys. Rev. Lett. 108, 201602 (2012).

[8] G. Policastro, D. T. Son, and A. O. Starinets, The Shear Viscosity of Strongly Coupled $N=4$ Supersymmetric Yang-Mills Plasma, Phys. Rev. Lett. 87, 081601 (2001).

[9] M. P. Heller, R. A. Janik, and P. Witaszczyk, Hydrodynamic Gradient Expansion in Gauge Theory Plasmas, Phys. Rev. Lett. 110, 211602 (2013)

[10] M. P. Heller and M. Spalinski, Hydrodynamics Beyond the Gradient Expansion: Resurgence and Resummation, Phys. Rev. Lett. 115, 072501 (2015).

[11] A. Buchel, M. P. Heller, and J. Noronha, Entropy production, hydrodynamics, and resurgence in the primordial quark-gluon plasma from holography, Phys. Rev. D 94, 106011 (2016).

[12] I. Müller, Zum Paradoxon der Wärmeleitungstheorie, Z. Phys. 198, 329 (1967).

[13] W. Israel and J. M. Stewart, Transient relativistic thermodynamics and kinetic theory, Ann. Phys. 118, 341 (1979).

[14] I. Aniceto and M. Spaliński, Resurgence in extended hydrodynamics, Phys. Rev. D 93, 085008 (2016).

[15] M. Strickland, J. Noronha, and G. S. Denicol, Anisotropic nonequilibrium hydrodynamic attractor, Phys. Rev. D 97, 036020 (2018).

[16] P. Romatschke, Relativistic Fluid Dynamics Far From Local Equilibrium, Phys. Rev. Lett. 120, 012301 (2018).

[17] M. Strickland, The non-equilibrium attractor for kinetic theory in relaxation time approximation, J. High Energy Phys. 12 (2018) 128.

[18] D. Almaalol, A. Kurkela, and M. Strickland, Nonequilibrium Attractor in High-Temperature QCD Plasmas, Phys. Rev. Lett. 125, 122302 (2020).

[19] G. S. Denicol and J. Noronha, Exact Hydrodynamic Attractor of an Ultrarelativistic Gas of Hard Spheres, Phys. Rev. Lett. 124, 152301 (2020).

[20] G. Basar and G. V. Dunne, Hydrodynamics, resurgence, and transasymptotics, Phys. Rev. D 92, 125011 (2015).

[21] M. P. Heller, A. Kurkela, M. Spaliński, and V. Svensson, Hydrodynamization in kinetic theory: Transient modes and the gradient expansion, Phys. Rev. D 97, 091503(R) (2018).
[22] M. P. Heller and V. Svensson, How does relativistic kinetic theory remember about initial conditions?, Phys. Rev. D 98, 054016 (2018).

[23] M. Attems, J. Casalderrey-Solana, D. Mateos, D. SantosOliván, C. F. Sopuerta, M. Triana, and M. Zilhão, Paths to equilibrium in non-conformal collisions, J. High Energy Phys. 06 (2017) 154.

[24] M. P. Heller, Holography, hydrodynamization and heavy-ion collisions, Acta Phys. Polon. B 47, 2581 (2016).

[25] A. Kurkela, W. van der Schee, U. A. Wiedemann, and B. Wu, Early- and Late-Time Behavior of Attractors in Heavy-Ion Collisions, Phys. Rev. Lett. 124, 102301 (2020).

[26] F. Gelis, E. Iancu, J. Jalilian-Marian, and R. Venugopalan, The color glass condensate, Annu. Rev. Nucl. Part. Sci. 60, 463 (2010).

[27] R. Baier, A. H. Mueller, D. Schiff, and D. T. Son, Bottom up thermalization in heavy ion collisions, Phys. Lett. B 502, 51 (2001).

[28] A. Kurkela and G. D. Moore, Bjorken flow, plasma instabilities, and thermalization, J. High Energy Phys. 11 (2011) 120.

[29] V. Balasubramanian, A. Bernamonti, J. de Boer, N. Copland, B. Craps, E. Keski-Vakkuri, B. Müller, A. Schäfer, M. Shigemori, and W. Staessens, Holographic thermalization, Phys. Rev. D 84, 026010 (2011).

[30] D. Steineder, S. A. Stricker, and A. Vuorinen, Holographic Thermalization at Intermediate Coupling, Phys. Rev. Lett. 110, 101601 (2013).

[31] S. A. Stricker, Holographic thermalization in $N=4$ super Yang-Mills theory at finite coupling, Eur. Phys. J. C 74, 2727 (2014).

[32] S. Waeber, A. Schäfer, A. Vuorinen, and L. G. Yaffe, Finite coupling corrections to holographic predictions for hot QCD, J. High Energy Phys. 11 (2015) 087.

[33] E. Iancu and A. Mukhopadhyay, A semi-holographic model for heavy-ion collisions, J. High Energy Phys. 06 (2015) 003.

[34] A. Mukhopadhyay, F. Preis, A. Rebhan, and S. A. Stricker, Semi-holography for heavy ion collisions: Self-consistency and first numerical tests, J. High Energy Phys. 05 (2016) 141.

[35] C. Ecker, A. Mukhopadhyay, F. Preis, A. Rebhan, and A. Soloviev, Time evolution of a toy semiholographic glasma, J. High Energy Phys. 08 (2018) 074.

[36] T. Faulkner and J. Polchinski, Semi-holographic Fermi liquids, J. High Energy Phys. 06 (2011) 012.

[37] A. Mukhopadhyay and G. Policastro, Phenomenological Characterization of Semiholographic Non-Fermi Liquids, Phys. Rev. Lett. 111, 221602 (2013).

[38] J. Casalderrey-Solana, D. C. Gulhan, J. G. Milhano, D. Pablos, and K. Rajagopal, A Hybrid Strong/Weak Coupling Approach to Jet Quenching, J. High Energy Phys. 10 (2014) 019; Erratum to: A hybrid strong/weak coupling approach to jet quenchin 09 (2015) 175.

[39] A. Kurkela, A. Mukhopadhyay, F. Preis, A. Rebhan, and A. Soloviev, Hybrid fluid models from mutual effective metric couplings, J. High Energy Phys. 08 (2018) 054.

[40] J. D. Bjorken, Highly relativistic nucleus-nucleus collisions: The central rapidity region, Phys. Rev. D 27, 140 (1983). 
[41] R. Baier, P. Romatschke, D. T. Son, A. O. Starinets, and M. A. Stephanov, Relativistic viscous hydrodynamics, conformal invariance, and holography, J. High Energy Phys. 04 (2008) 100.

[42] S. Banerjee, N. Gaddam, and A. Mukhopadhyay, Illustrated study of the semiholographic nonperturbative framework, Phys. Rev. D 95, 066017 (2017).

[43] G. S. Denicol and J. Noronha, Analytical attractor and the divergence of the slow-roll expansion in relativistic hydrodynamics, Phys. Rev. D 97, 056021 (2018).

[44] C. Loizides, Experimental overview on small collision systems at the LHC, Nucl. Phys. A 956, 200 (2016).
[45] C. Shen, J.-F. Paquet, G. S. Denicol, S. Jeon, and C. Gale, Collectivity and electromagnetic radiation in small systems, Phys. Rev. C 95, 014906 (2017).

[46] S. Schlichting and P. Tribedy, Collectivity in small collision systems: An initial-state perspective, Adv. High Energy Phys. 2016, 8460349.

[47] P. M. Chesler, Colliding Shock Waves and Hydrodynamics in Small Systems, Phys. Rev. Lett. 115, 241602 (2015).

[48] M. P. Heller, R. Jefferson, M. Spalinski, and V. Svensson, Hydrodynamic Attractors in Phase Space, Phys. Rev. Lett. 125, 132301 (2020) 